DSF-T-99/46

\title{
Heavy neutrino mass scale and quark-lepton symmetry
}

\author{
D. Falcone \\ Dipartimento di Scienze Fisiche, Università di Napoli, \\ Mostra d'Oltremare, Pad. 19, I-80125, Napoli, Italy \\ e-mail: falcone@na.infn.it
}

\begin{abstract}
Assuming hierarchical neutrino masses we calculate the heavy neutrino mass scale in the seesaw mechanism from experimental data on oscillations of solar and atmospheric neutrinos and quark-lepton symmetry. The resulting scale is around or above the unification scale, unless the two lightest neutrinos have masses of opposite sign, in which case the resulting scale can be intermediate.
\end{abstract}

PACS: 12.15.Ff, 14.60.Pq

Keywords: Neutrino Physics; Grand Unified Theories 
Recent results on atmospheric and solar neutrinos [1] support the idea that neutrinos have tiny masses. A popular mechanism for achieving small Majorana masses for left-handed neutrinos is the seesaw mechanism [2], where the Majorana mass matrix of the left-handed neutrino is given by

$$
M_{L}=M_{D} M_{R}^{-1} M_{D}^{T}
$$

with $M_{D}$ the Dirac mass matrix and $M_{R}$ the Majorana mass matrix of a heavy right-handed neutrino, related to lepton number violation at high energy [3]. If the Dirac masses are supposed to be of the same order of magnitude of the up-quark masses (quark-lepton symmetry), as suggested by GUTs [4], then light left-handed neutrinos appear. In such a case the heavy neutrino mass scale should be at the unification or intermediate scale [3], because in most GUTs the Higgs field that gives mass to neutrinos is the same that breaks the GUT group or the intermediate group to the standard model. Also, at that scale the relation $m_{b}=m_{\tau}$, resulting from quark-lepton symmetry, should hold.

In this short paper the question of calculating the heavy neutrino mass scale from experimental data and quark-lepton symmetry is addressed [5] 7], assuming $\left|m_{3}\right| \gg\left|m_{1,2}\right|$, where $m_{i}$ are the light neutrino masses, and it is shown that such a scale is at or above the unification scale, unless $m_{1} \simeq-m_{2}$. For nearly opposite $m_{1}$, $m_{2}$, the heavy neutrino mass scale is around the intermediate scale of a GUT such as $S O(10)$ with $S U(4) \times S U(2) \times S U(2)$ as intermediate symmetry [8].

Assuming maximal mixing for atmospheric neutrinos and that the electron neutrino does not contribute to the atmospheric oscillations [9], the matrix $M_{L}$ can be written as [10]

$$
M_{L}=\left(\begin{array}{ccc}
2 \epsilon & \delta & \delta \\
\delta & \sigma & \rho \\
\delta & \rho & \sigma
\end{array}\right)
$$


with

$$
\begin{aligned}
\epsilon & =\frac{1}{2}\left(m_{1} c^{2}+m_{2} s^{2}\right) \\
\delta & =\frac{1}{\sqrt{2}}\left(m_{1}-m_{2}\right) c s \\
\sigma & =\epsilon_{2}+\frac{m_{3}}{2} \\
\rho & =\epsilon_{2}-\frac{m_{3}}{2} \\
\epsilon_{2} & =\frac{1}{2}\left(m_{1} s^{2}+m_{2} c^{2}\right),
\end{aligned}
$$

and $c=\cos \theta, s=\sin \theta$, where $\theta$ is the mixing angle of solar neutrinos. The inverse of $M_{L}$ is given by

$$
M_{L}^{-1}=\left(\begin{array}{ccc}
\sigma^{2}-\rho^{2} & \delta(\rho-\sigma) & \delta(\rho-\sigma) \\
\delta(\rho-\sigma) & 2 \epsilon \sigma-\delta^{2} & \delta^{2}-2 \epsilon \rho \\
\delta(\rho-\sigma) & \delta^{2}-2 \epsilon \rho & 2 \epsilon \sigma-\delta^{2}
\end{array}\right) \frac{1}{m_{1} m_{2} m_{3}}
$$

Without loss of generality we can assume $m_{3}>0$. Moreover, if $m_{3} \gg\left|m_{2}\right|,\left|m_{1}\right|$, with $\Delta m_{\text {sun }}^{2}=m_{2}^{2}-m_{1}^{2}$ and $\Delta m_{\text {atm }}^{2}=m_{3}^{2}-m_{1,2}^{2}$, then

$$
M_{L}^{-1}=\left(\begin{array}{ccc}
0 & -\delta & -\delta \\
-\delta & \epsilon & \epsilon \\
-\delta & \epsilon & \epsilon
\end{array}\right) \frac{1}{m_{1} m_{2}}
$$

In the basis where the charged lepton mass matrix $M_{e}$ is diagonal, for $M_{D}$ we take

$$
M_{D}=\frac{m_{\tau}}{m_{b}} \operatorname{diag}\left(m_{u}, m_{c}, m_{t}\right)
$$

An almost diagonal form of this kind for $M_{D}$ is again suggested by quark-lepton symmetry and GUTs [11], and the factor is due to running from unification or intermediate scale [12], corresponding to supersymmetric and nonsupersymmetric cases, respectively. From the seesaw formula (1) and eqn.(10) we obtain

$$
M_{R}=M_{D} M_{L}^{-1} M_{D}
$$


and, due to the form of $M_{L}^{-1}$ and $M_{D}$ in eqns.(9),(10), two elements of $M_{R}$ have to be considered to discover the scale of heavy neutrino mass:

$$
\begin{aligned}
& M_{R 33}=k \frac{m_{1} c^{2}+m_{2} s^{2}}{m_{1} m_{2}} m_{t}^{2}, \\
& M_{R 13}=k \frac{1}{\sqrt{2}} \frac{\left(m_{1}-m_{2}\right) c s}{m_{1} m_{2}} m_{u} m_{t}
\end{aligned}
$$

with $k=\left(m_{\tau} / m_{b}\right)^{2}$. We will consider two cases for $s$, namely $s=0$ (single maximal mixing) and $s=1 / \sqrt{2}$ (bimaximal mixing [13]). For $s=0$ we get the scale

$$
M_{R 33}=k \frac{m_{t}^{2}}{m_{2}}
$$

For $s=1 / \sqrt{2}$ we have three subcases: $\left|m_{2}\right| \gg\left|m_{1}\right|$ which gives

$$
M_{R 33}=k \frac{1}{2} \frac{m_{t}^{2}}{m_{1}}
$$

$m_{2} \simeq m_{1}$ yielding

$$
M_{R 33}=k \frac{m_{t}^{2}}{m_{1,2}} ;
$$

and $m_{2} \simeq-m_{1}$ for which the scale is given by

$$
M_{R 13}=k \frac{1}{\sqrt{2}} \frac{m_{u} m_{t}}{m_{1,2}}
$$

while $M_{R 33}$ is much smaller. The case $s=0$ is near the small mixing MSW solution of the solar neutrino problem [14], while the case $s=1 / \sqrt{2}$ is near both the large mixing MSW and especially the vacuum oscillations [14]. Using the same numerical values of ref. [6] we find $M_{R 33} \gtrsim 10^{15} \mathrm{GeV}$ for $s=0$. For $s=1 / \sqrt{2}$, the three eqns.(15),(16) and (17) lead respectively to: $M_{R 33} \gtrsim 10^{16} \mathrm{GeV}$ (large mixing MSW), $M_{R 33} \gtrsim 10^{18}$ $\mathrm{GeV}$ (vacuum oscillations); $M_{R 33} \gtrsim 10^{15} \mathrm{GeV}$ (LMSW and VO); $M_{R 13} \gtrsim 10^{10} \mathrm{GeV}$ (LMSW and VO). Therefore we find that the heavy neutrino mass scale can be at the intermediate scale only if $m_{2} \simeq-m_{1}$ (see ref. [15]), and $M_{R}$ has a roughly off-diagonal form. As the intermediate scale is related to the nonsupersymmetric case [16], we get that nearly opposite masses are also related to that case (we are 
in a $\mathrm{CP}$ conserving framework, the mass sign corresponds to $\mathrm{CP}$ parity [14]). For positive masses the two MSW solutions are in agreement with the unification scale while the vacuum oscillation solution gives a scale well above the unification scale, unless $m_{2} \simeq m_{1}$. It is worth noting that a smaller mass is obtained for opposite $m_{1}$, $m_{2}$, due to the appearance of $m_{u} m_{t}$, rather than $m_{t}^{2}$, in eqn.(17). Moreover, from eqns.(14-17) one can read the dependence of the heavy neutrino mass scale on light neutrino masses. This dependence is in agreement with the analyses given in refs. [6, [], where only positive values of the light neutrino masses are considered. The three eigenvalues of $M_{R}$ have a strong hierarchy, unless there is one negative mass, in which case the structure of $M_{R}$ is very different from that of $M_{D}$. Finally, we notice that the bimaximal case with opposite masses has a different impact on the neutrinoless double-beta decay parameter $\mathcal{M}_{e e}=2 \epsilon$, with respect to the bimaximal case with positive masses and that, for a hierarchical spectrum, neutrinos are not relevant for hot dark matter.

In conclusion, we have calculated the scale of heavy neutrino mass in the seesaw mechanism assuming hierarchical light neutrino masses, maximal or bimaximal mixing (as suggested by experimental data) and quark-lepton symmetry. The main results are: intermediate scale is obtained only if the two lightest left-handed neutrinos have nearly opposite masses; the vacuum oscillation case with positive and full hierarchical masses leads to a scale near the Planck mass. 
[1] Neutrino 98, Nucl. Phys. B (Proc. Suppl.) 77 (1999) 1

[2] M. Gell-Mann, P. Ramond and R. Slansky, in Supergravity, eds. P. van Nieuwenhuizen and D. Freedman (North Holland, Amsterdam, 1979)

T. Yanagida, in Proceedings of the Workshop on Unified Theory and Baryon Number in the Universe, eds. O. Sawada and A. Sugamoto (KEK, 1979)

S. Weinberg, Phys. Rev. Lett. 43 (1979) 1566

R.N. Mohapatra and G. Senjanovic, Phys. Rev. Lett. 44 (1980) 912

[3] R.N. Mohapatra and P.B. Pal, Massive Neutrinos in Physics and Astrophysics (World Scientific, Singapore, 1991)

[4] See, for SO(10), J. Harvey, D. Reiss and P. Ramond, Nucl. Phys. B 199 (1982) 223

[5] A. Yu. Smirnov, Nucl. Phys. B 466 (1996) 25

[6] D. Falcone, hep-ph/9909207 (Phys. Rev. D, to be published)

[7] T.K. Kuo, G.-H. Wu and S.W. Mansour, hep-ph/9912366

[8] N.G. Deshpande, E. Keith and P.B. Pal, Phys. Rev. D 46 (1992) 2261

D.-G. Lee, R.N. Mohapatra, M.K. Parida and M. Rani, Phys. Rev. D 51 (1995) 229

O. Pisanti and L. Rosa, Prog. Part. Nucl. Phys. 40 (1998) 81

[9] Y. Fukuda et al. (SuperKamiokande Collaboration), Phys. Rev. Lett. 81 (1998) 1562 K. Scholberg (SuperKamiokande Collaboration), hep-ex/9905016

M. Apollonio et al. (CHOOZ Collaboration), Phys. Lett. B 420 (1998) 397

[10] G. Altarelli and F. Feruglio, Phys. Rep. 320 (1999) 295

[11] P. Ramond, R.G. Roberts and G.G. Ross, Nucl. Phys. B 406 (1993) 19 
[12] H. Arason, D.J. Castano, E.J. Piard and P. Ramond, Phys. Rev. D 47 (1993) 232

[13] V. Barger, S. Pakwasa, T.J. Weiler and K. Whisnant, Phys. Lett. B 437 (1998) 107 A.T. Baltz, A.S. Goldhaber and M. Goldhaber, Phys. Rev. Lett. 81 (1998) 5730 F. Vissani, hep-ph/9708483

[14] S.M. Bilenky, C. Giunti and W. Grimus, Prog. Part. Nucl. Phys. 43 (1999) 1

[15] M. Jezabek and Y. Sumino, Phys. Lett. B 440 (1998) 327; 457 (1999) 139 B. Stech, Phys. Lett. B 465 (1999) 219

M. Abud, F. Buccella, D. Falcone, G. Ricciardi and F. Tramontano, hep-ph/9911238 E.Kh. Akhmedov, G.C. Branco and M.N. Rebelo, hep-ph/9911364

[16] See for example N.G. Deshpande and E. Keith, Phys. Rev. D 50 (1994) 3513 